\title{
Study on Zero-Doppler Centroid Control for GEO SAR Ground Observation
}

\author{
Yicheng Jiang, Bin Hu, Yun Zhang, Meng Lian, and Zhuoqun Wang \\ Research Institute of Electronic Engineering Technology, Harbin Institute of Technology, No. 714, Harbin 150001, China \\ Correspondence should be addressed to Bin Hu; hubin_hit@qq.com
}

Received 7 March 2014; Revised 11 June 2014; Accepted 8 July 2014; Published 26 November 2014

Academic Editor: Bing Liu

Copyright (C) 2014 Yicheng Jiang et al. This is an open access article distributed under the Creative Commons Attribution License, which permits unrestricted use, distribution, and reproduction in any medium, provided the original work is properly cited.

In geosynchronous Earth orbit SAR (GEO SAR), Doppler centroid compensation is a key step for imaging process, which could be performed by the attitude steering of a satellite platform. However, this zero-Doppler centroid control method does not work well when the look angle of radar is out of an expected range. This paper primarily analyzes the Doppler properties of GEO SAR in the Earth rectangular coordinate. Then, according to the actual conditions of the GEO SAR ground observation, the effective range is presented by the minimum and maximum possible look angles which are directly related to the orbital parameters. Based on the vector analysis, a new approach for zero-Doppler centroid control in GEO SAR, performing the attitude steering by a combination of pitch and roll rotation, is put forward. This approach, considering the Earth's rotation and elliptical orbit effects, can accurately reduce the residual Doppler centroid. All the simulation results verify the correctness of the range of look angle and the proposed steering method.

\section{Introduction}

There has been an increasing interest in Geosynchronous Earth orbit SAR (GEO SAR) for the purpose of surveillance with large observation. In fact, this broadside-looking SAR has a non-zero-Doppler centroid due to the Earth's rotation and elliptical orbit effects [1]. Compensating the residual Doppler centroid is an important step to solve the rangeazimuth coupling which decreases the quality of SAR imaging. The zero-Doppler attitude steering method was first put forward by Raney in the literature [2], which used a 1D yaw steering method to compensate the Doppler shift induced by the Earth's rotation. This method is useful in circular orbits, but it causes larger Doppler shifts in elliptical satellite orbits like GEO SAR. Later, a method (the TerraSAR-X method) of $2 \mathrm{D}$ attitude steering was carried out in the low Earth elliptical orbits (LEO) SAR in literatures $[3,4]$, yet this $2 \mathrm{D}$ attitude steering method does not work well particularly with the large residual Doppler centroid exited in GEO SAR. Then, the total zero-Doppler steering (TZDS) method was proposed in the literatures $[5,6]$, taking the Earth's rotation and elliptical orbit effects into account. The method in [5] can minimize the Doppler residuals in the LEO SAR system, but it is not suitable for the GEO SAR system. Subsequently, a 2D phase scan method was introduced in literatures $[7,8]$, which carried out a highly accurate compensation of the Doppler centroid in GEO SAR. By using the 2D phased scan instead of the attitude steering, the approach in [7] can avoid the satellite platform rotation and stabilization.

However, these methods mentioned above are invalid or inaccurate when the look angle of radar is out of an effective range, which is elaborated as follows.

(i) The Earth's surface could not be observed by the radar when the look angle is greater than the maximum of the range.

(ii) The coverage of radar cannot provide valid echoes with a zero-Doppler centroid when the look angle is less than the minimum of the range.

For the above issues, this paper introduces a general approach to compensate the Doppler centroid frequency for the spaceborne SAR which works with a look angle within an effective range. The paper is organized as follows. Section 2 describes the GEO SAR geometry and notation, and the Doppler vector of the satellite-borne SAR is analyzed in the Earth rectangular coordinate (ERC). In Section 3, the derivation of the effective range of the look angle is firstly 


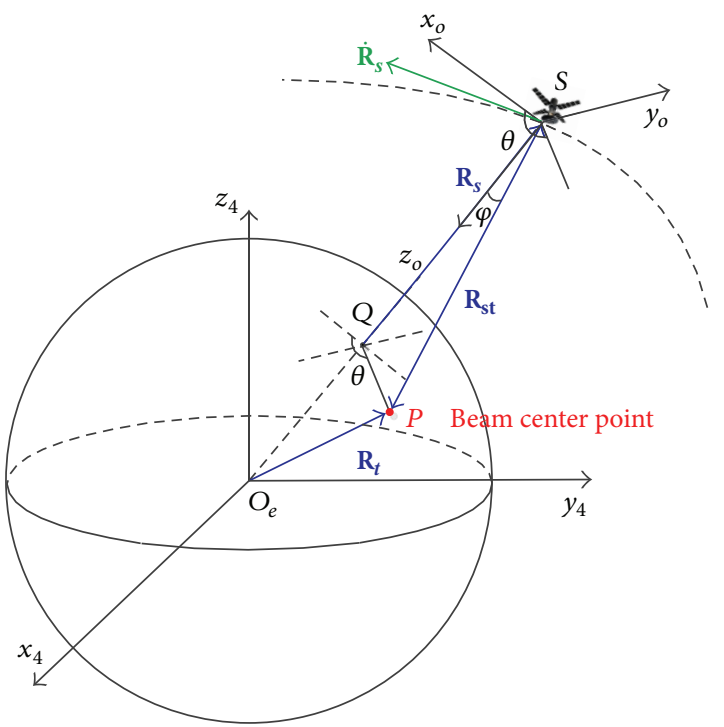

Figure 1: Geometry of the earth rectangular coordinates and satellite local coordinates.

presented in detail. The range is determined by the satellite orbit parameters such as the orbital eccentricity, the argument of perigee. Then, based on a reasonable look angle, a new method of zero-Doppler centroid control, performed by the pitch and roll attitude steering, is introduced. In Section 4, simulation results validate the correctness of derivation in the paper. Conclusions appear in Section 5.

\section{Vector Analysis in GEO SAR Model}

The geometry of the Earth rectangular coordinate (ERC) $\mathrm{O}_{e}-x_{4} y_{4} z_{4}$ is shown in Figure 1. The ERC can be defined as the coordinate origin is the Earth's center $O_{e}$, the $O_{e} x_{4}$-axis is along the Greenwich meridian direction in the equatorial plane, the axis of $\mathrm{O}_{e} z_{4}$-axis is along the Earth angular momentum direction, and the $\mathrm{O}_{e} y_{4}$-axis obeys a right-hand rule. In Figure 1, $P$ is the intersection of the beam-pointing direction and the Earth's surface, which is called the "beam center point" (BCP) in this paper, and $Q$ is the nadir point of the satellite. $\mathbf{R}_{\mathbf{t}}$ and $\mathbf{R}_{\mathbf{s}}$ are the position vectors of the BCP and the satellite, respectively. $\dot{\mathbf{R}}_{\mathbf{s}}$ and $\dot{\mathbf{R}}_{\mathbf{t}}$ are the first-order time derivative of $\mathbf{R}_{\mathbf{s}}$ and $\mathbf{R}_{\mathbf{t}}$. The relative position vector between the satellite and the BCP is expressed as $\mathbf{R}_{\mathbf{s t}}=\mathbf{R}_{\mathbf{s}}-\mathbf{R}_{\mathbf{t}}$. The slant range $R_{\mathrm{st}}=\left|\mathbf{R}_{\mathrm{s}}-\mathbf{R}_{\mathbf{t}}\right|$ varies along the orbit, which can be calculated by

$$
R_{\mathrm{st}}(t)=R_{s}(t) \cos (\varphi)-\sqrt{R_{t}^{2}-R_{s}^{2}(t) \sin (\varphi)}
$$

where $\varphi$ is the look angle of radar (shown in Figure 1); $R_{s}(t)$ and $R_{t}$ are the magnitudes of $\mathbf{R}_{\mathbf{s}}(t)$ and $\mathbf{R}_{\mathbf{t}}(t)$, respectively. Note that $R_{t}$ is a constant that is the length of the Earth's radius. The formula of $R_{s}(t)$ is given by the literature [9].
The Doppler centroid $f_{\mathrm{dc}}$ of the beam center point along the orbit is given by the literature [7]:

$$
\begin{aligned}
f_{\mathrm{dc}}(t) & =-\frac{2}{\lambda} \frac{\mathbf{R}_{\mathbf{s t}}(t) \cdot \dot{\mathbf{R}}_{\mathbf{s t}}(t)}{\left|\mathbf{R}_{\mathbf{s t}}(t)\right|} \\
& =-\frac{2}{\lambda} \frac{\left(\mathbf{R}_{\mathbf{s}}(t)-\mathbf{R}_{\mathbf{t}}(t)\right) \cdot\left(\dot{\mathbf{R}}_{\mathbf{s}}(t)-\dot{\mathbf{R}}_{\mathbf{t}}(t)\right)}{\left|\mathbf{R}_{\mathbf{s}}(t)-\mathbf{R}_{\mathbf{t}}(t)\right|},
\end{aligned}
$$

where $\lambda$ is the radar wavelength; the symbol \| indicates the vector magnitude. Noting that $\dot{\mathbf{R}}_{\mathbf{t}}(t)=\mathbf{0}$ in the ERC, the formula of the Doppler centroid can be rewritten as

$$
f_{\mathrm{dc}}(t)=-\frac{2}{\lambda} \frac{\left(\mathbf{R}_{\mathbf{s}}(t)-\mathbf{R}_{\mathbf{t}}(t)\right) \cdot \dot{\mathbf{R}}_{\mathbf{s}}(t)}{\left|\mathbf{R}_{\mathbf{s}}(t)-\mathbf{R}_{\mathbf{t}}(t)\right|} .
$$

It is apparent that the Doppler centroid $f_{\mathrm{dc}}(t)$ is not only determined by the satellite position, but also dependent on the position of the BCP. Both are important for the analytical derivation of the $2 \mathrm{D}$ attitude steering angles, which will be shown in Section 3.

To obtain the analytic formula for the position vector of the BCP, it is needed to find the relationship between the satellite and the BCP in the satellite local coordinate system (SCS) $S-x_{o} y_{o} z_{o}$ (see Figure 1). The SCS is defined as the coordinate origin is the center of the satellite mass, the $S z_{o}$-axis is along the satellite position vector towards the Earth's center, the axis of $S x_{o}$ is perpendicular to the $S z_{o}$-axis in the orbital plane (the angle between the $S x_{o}$ axis and the satellite velocity vector is less than $90^{\circ}$ ), and the $S y_{o}$-axis obeys the right-hand rule. The formula for the slant range vector of the BCP in the SCS can be expressed as

$$
\begin{aligned}
\mathbf{R}_{\mathrm{st}}(t)=\left[R_{\mathrm{st}}(t) \sin (\varphi) \cos (\theta(t))\right. \\
\left.\quad-R_{\mathrm{st}}(t) \sin (\varphi) \sin (\theta(t)) R_{\mathrm{st}}(t) \cos (\varphi)\right]^{T},
\end{aligned}
$$

where $\theta(t)$ is the angle between the axis $S x_{o}$ and the projection of the beam pointing vector in the $x_{o}-y_{o}$ plane (as shown in Figure 1) and can be presented as

$$
\theta(t)=\arccos \left(\frac{\sin \left(\theta_{s}\right)}{\cos (\psi(t))}\right)
$$

where $\theta_{s}$ is the squint angle of radar and $\psi(t)$ is the angle between the $S x_{o}$-axis and the satellite velocity vector:

$$
\psi(t)=\arccos \left(\frac{\mathbf{R}_{\mathbf{s}}(t) \cdot \dot{\mathbf{R}}_{\mathbf{s}}(t)}{\left|\mathbf{R}_{\mathbf{s}}(t)\right| \cdot\left|\dot{\mathbf{R}}_{\mathbf{s}}(t)\right|}\right)-\frac{\pi}{2} .
$$

The position equation of the BCP in the Earth rectangular coordinate is given by

$$
\mathbf{R}_{\mathbf{t}}(t)=\left[\begin{array}{c}
X_{\mathbf{t}}(t) \\
Y_{\mathbf{t}}(t) \\
Z_{\mathbf{t}}(t)
\end{array}\right]=\mathbf{W}_{\mathbf{A}}(t) \times \mathbf{W}_{\mathbf{B}} \times \mathbf{W}_{\mathbf{c}}(t) \times \mathbf{R}_{\mathbf{s p}}(t)
$$


where

$$
\begin{gathered}
\mathbf{W}_{\mathbf{A}}(t)=\left[\begin{array}{ccc}
\cos \left(w_{e} \cdot t\right) & \sin \left(w_{e} \cdot t\right) & 0 \\
-\sin \left(w_{e} \cdot t\right) & \cos \left(w_{e} \cdot t\right) & 0 \\
0 & 0 & 1
\end{array}\right], \\
\mathbf{W}_{\mathbf{B}}=\left[\begin{array}{ccc}
\cos \left(\Omega_{0}\right) & -\sin \left(\Omega_{0}\right) & 0 \\
\sin \left(\Omega_{0}\right) & \cos \left(\Omega_{0}\right) & 0 \\
0 & 0 & 1
\end{array}\right] \times\left[\begin{array}{ccc}
1 & 0 & 0 \\
0 & \cos (i) & \sin (i) \\
0 & -\sin (i) & \cos (i)
\end{array}\right] \\
\times\left[\begin{array}{ccc}
\cos \left(\omega_{0}\right) & -\sin \left(\omega_{0}\right) & 0 \\
\sin \left(\omega_{0}\right) & \cos \left(\omega_{0}\right) & 0 \\
0 & 0 & 1
\end{array}\right], \\
\mathbf{W}_{\mathbf{c}}(t)=\left[\begin{array}{ccc}
\cos (f(t)) & -\sin (f(t)) & 0 \\
\sin (f(t)) & \cos (f(t)) & 0 \\
0 & 0
\end{array}\right], \\
\mathbf{R}_{\mathbf{s p}}(t)=\left[\begin{array}{ccc}
0 & 0 & -1 \\
1 & 0 & 0 \\
0 & -1 & 0
\end{array}\right] \times \mathbf{R}_{\mathbf{s t}}(t)+\left[\begin{array}{c}
R_{s}(t) \\
0 \\
0
\end{array}\right],
\end{gathered}
$$

where the symbol $\times$ represents cross product, $w_{e}$ is the Earth's angular velocity, $i$ indicates the orbit inclination, $\Omega_{0}$ is the longitude of ascending node, $\omega_{0}$ represents the argument of perigee, and $f(t)$ indicates the argument of latitude (i.e., the sum of the true anomaly and the perigee argument).

\section{Zero-Doppler Centroid Control}

As we known, when the angle $\theta=90^{\circ}$, the beam-pointing vector is perpendicular to the velocity vector of satellite, and $\mathbf{R}_{\mathbf{s t}}(t) \cdot \dot{\mathbf{R}}_{\mathbf{s t}}(t)=0$ in (2). However, owing to the influences of earth rotation and elliptical orbit, sometimes $\mathbf{R}_{\mathbf{s t}}(t) \cdot \dot{\mathbf{R}}_{\mathbf{s t}}(t) \neq 0$, especially in GEO SAR, and it affects the quality of image by the residual Doppler centroid. Fortunately, it can be resolved by steering the satellite's attitude angles to reduce the offset of the Doppler centroid.

The geometry of the spaceborne radar, after zero-Doppler centroid control, is illustrated in Figure 2, in which the radar observes the Earth's surface with the look angle $\varphi . \alpha$ is the angle of instance; $\beta$ is the geocentric angle which is given by

$$
\beta(t)=\arcsin \left[\frac{R_{s}(t)}{R_{t}} \sin (\varphi)\right]-\varphi .
$$

The distance between the radar and the BCP over the orbital period can be calculated by

$$
\begin{aligned}
\left|\mathbf{R}_{\mathbf{s t}}(t)\right|^{2}= & \left|\mathbf{R}_{\mathbf{s}}(t)\right|^{2}+\left|\mathbf{R}_{\mathbf{t}}(t)\right|^{2} \\
& -2\left|\mathbf{R}_{\mathbf{s}}(t)\right|\left|\mathbf{R}_{\mathbf{t}}(t)\right| \cos (\beta(t)) .
\end{aligned}
$$

After the attitude steering, the relative velocity vector between the satellite and the BCP is perpendicular to the relative position vector. According to the properties of the vector inner product, we have

$$
\mathbf{R}_{\mathbf{s t}}(t) \cdot \dot{\mathbf{R}}_{\mathbf{s}}(t)=0 .
$$

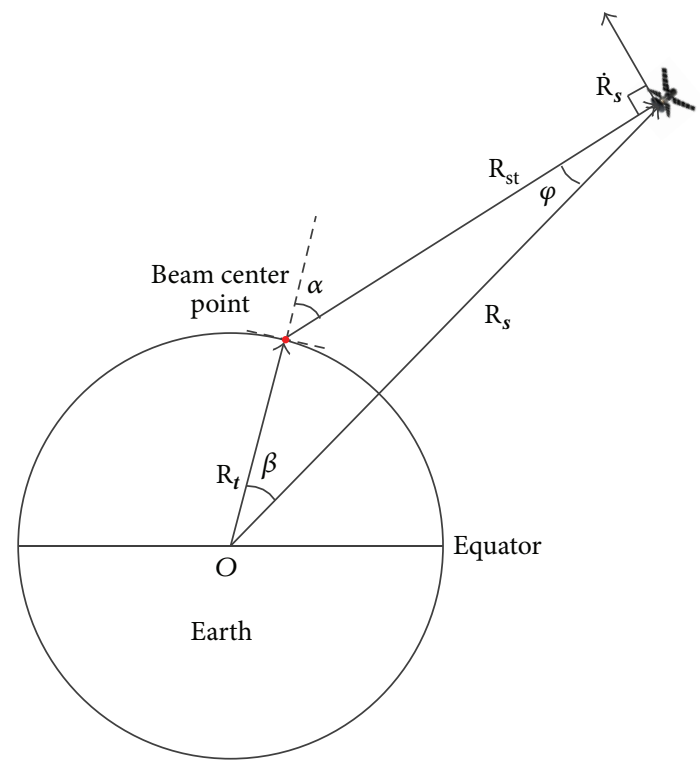

FIGURE 2: Geometry of the satellite after zero-Doppler centroid control.

Then, combining the aforementioned expressions, the position equation of the new beam center point (after the attitude steering) in ERC, which is expressed as $[x(t), y(t), z(t)]$ for the convenience of clarity, can be calculated by the following equations:

$$
\begin{gathered}
x^{2}(t)+y^{2}(t)+z^{2}(t)=R_{t}^{2}, \\
\left(x(t)-x_{s}(t)\right)^{2}+\left(y(t)-y_{s}(t)\right)^{2}+\left(z(t)-z_{s}(t)\right)^{2} \\
=R_{s}^{2}(t)+R_{t}^{2}-2 R_{s}(t) R_{t} \cos (\beta(t)) \\
\left(x(t)-x_{s}(t)\right) \dot{x}_{s}(t)+\left(y(t)-y_{s}(t)\right) \dot{y}_{s}(t) \\
+\left(z(t)-z_{s}(t)\right) \dot{z}_{s}(t)=0
\end{gathered}
$$

where the position vector of the satellite $\left[x_{s}(t), y_{s}(t), z_{s}(t)\right]$ and its velocity vector $\left[\dot{x}_{s}(t), \dot{y}_{s}(t), \dot{z}_{s}(t)\right]$ are given by the literature [10]. $x(t), y(t)$, and $z(t)$ are solved as follows:

$$
\begin{aligned}
x(t)= & \frac{B(t) x_{s}(t)}{R_{s}^{2}(t)} \\
& +\frac{R_{s}^{2}(t) \dot{x}_{s}^{2}(t) A(t)-x_{s}(t) A^{2}(t)-\dot{x}_{s}(t) A(t) B(t)}{R_{s}^{2}(t)\left(\dot{x}_{s}^{2}(t)+\dot{y}_{s}^{2}(t)+\dot{z}_{s}^{2}(t)\right)} \\
& +k \frac{y_{s}(t) \dot{z}_{s}(t)-\dot{y}_{s}(t) z_{s}(t)}{R_{s}^{2}(t)} C(t),
\end{aligned}
$$




$$
\begin{aligned}
y(t)= & \frac{B(t) y_{s}(t)}{R_{s}^{2}(t)} \\
& +\frac{R_{s}^{2}(t) \dot{y}_{s}^{2}(t) A(t)-y_{s}(t) A^{2}(t)-\dot{y}_{s}(t) A(t) B(t)}{R_{s}^{2}(t)\left(\dot{x}_{s}^{2}(t)+\dot{y}_{s}^{2}(t)+\dot{z}_{s}^{2}(t)\right)} \\
& +k \frac{z_{s}(t) \dot{x}_{s}(t)-\dot{z}_{s}(t) x_{s}(t)}{R_{s}^{2}(t)} C(t), \\
z(t)= & \frac{B(t) z_{s}(t)}{R_{s}^{2}(t)} \\
& +\frac{R_{s}^{2}(t) \dot{z}_{s}^{2}(t) A(t)-z_{s}(t) A^{2}(t)-\dot{z}_{s}(t) A(t) B(t)}{R_{s}^{2}(t)\left(\dot{x}_{s}^{2}(t)+\dot{y}_{s}^{2}(t)+\dot{z}_{s}^{2}(t)\right)} \\
& +k \frac{x_{s}(t) \dot{y}_{s}(t)-y_{s}(t) \dot{x}_{s}(t)}{R_{s}^{2}(t)} C(t),
\end{aligned}
$$

where

$$
\begin{gathered}
A(t)=x_{s}(t) \dot{x}_{s}(t)+y_{s}(t) \dot{y}_{s}(t)+z_{s}(t) \dot{z}_{s}(t) \\
B(t)=R_{s}(t) R_{t} \cos (\beta(t))
\end{gathered}
$$

$C(t)$

$$
=\sqrt{R_{s}^{2}(t) R_{t}^{2} \sin ^{2}(\beta(t))-\frac{A^{2}(t)\left(R_{t}^{2}+R_{s}(t)^{2}-2 B(t)\right)}{\left(\dot{x}_{s}^{2}(t)+\dot{y}_{s}^{2}(t)+\dot{z}_{s}^{2}(t)\right)^{2}}} .
$$

In (13), $k=+1$ denotes the radar looks from the left-side, while $k=-1$ means the radar is right looking.

Under the condition of circular orbit, $A(t)=0$ and (13) can have solutions. For an elliptical orbit SAR, like GEO SAR, the necessary and sufficient condition when (13) has solutions is that the square root terms in $C(t)$ need to meet the following requirements:

$$
\begin{aligned}
R_{s}^{2}(t) & R_{t}^{2} \sin ^{2}(\beta(t)) \\
& -\frac{A^{2}(t)\left(R_{t}^{2}+R_{s}(t)^{2}-2 B(t)\right)}{\left(\dot{x}_{s}^{2}(t)+\dot{y}_{s}^{2}(t)+\dot{z}_{s}^{2}(t)\right)^{2}} \geq 0 .
\end{aligned}
$$

Meanwhile, to ensure the observation of a target fixed on the Earth by a space-borne SAR, the following inequality need to be satisfied:

$$
-\arcsin \left(\frac{R_{t}}{R_{s}(t)}\right) \leq \phi(t) \leq \arcsin \left(\frac{R_{t}}{R_{s}(t)}\right) .
$$

Combining (15) and (16), without the loss of generality, the range of the look angle is expressed as

$$
\arcsin \left(\frac{|A(t)|}{R_{s}(t)\left|\dot{\mathbf{R}}_{\mathbf{s}}(t)\right|}\right) \leq|\varphi(t)| \leq \arcsin \left(\frac{R_{t}}{R_{s}(t)}\right) .
$$

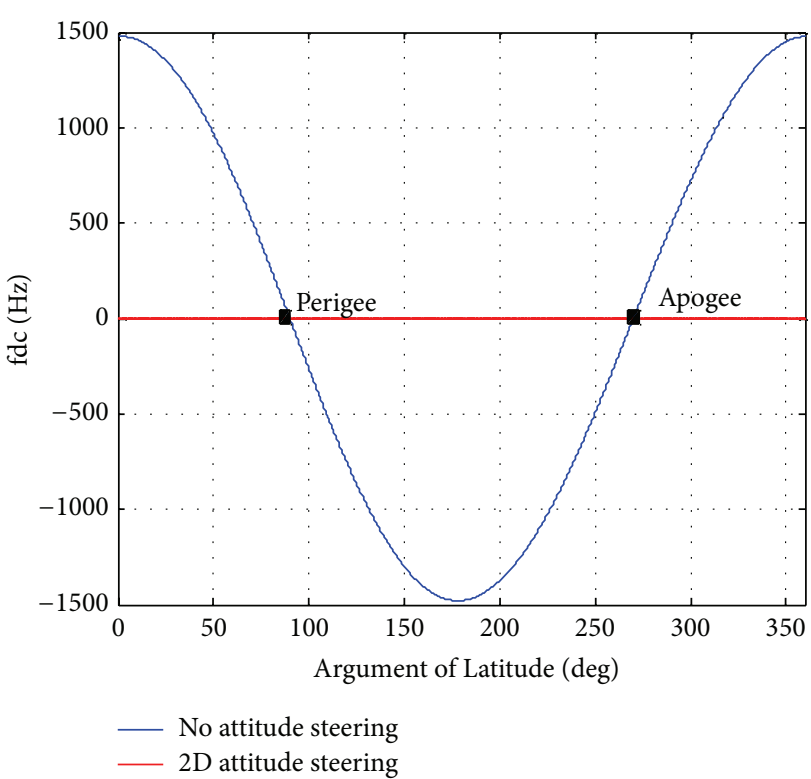

Figure 3: Comparison of the Doppler residuals before and after the attitude steering.

Through the transformation matrix between the SCS and the ERC, the track of the new beam center point in the SCS can be calculated by

$$
\begin{aligned}
{\left[\begin{array}{l}
x_{0}(t) \\
y_{0}(t) \\
z_{0}(t)
\end{array}\right]=} & {\left[\begin{array}{ccc}
0 & 1 & 0 \\
0 & 0 & -1 \\
-1 & 0 & 0
\end{array}\right] \times \mathbf{U}_{\mathbf{A}}(t) \times \mathbf{U}_{\mathbf{B}} \times \mathbf{U}_{\mathbf{C}}(t) } \\
& \times\left[\begin{array}{c}
x(t) \\
y(t) \\
z(t)
\end{array}\right]+\left[\begin{array}{c}
0 \\
0 \\
r(t)
\end{array}\right],
\end{aligned}
$$

where

$$
\begin{gathered}
\mathbf{U}_{\mathbf{A}}(t)=\left[\begin{array}{ccc}
\cos (f(t)) & \sin (f(t)) & 0 \\
-\sin (f(t)) & \cos (f(t)) & 0 \\
0 & 0 & 1
\end{array}\right], \\
\mathbf{U}_{\mathbf{B}}=\left[\begin{array}{ccc}
\cos \left(\omega_{0}\right) & \sin \left(\omega_{0}\right) & 0 \\
-\sin \left(\omega_{0}\right) & \cos \left(\omega_{0}\right) & 0 \\
0 & 0 & 1
\end{array}\right] \\
\times\left[\begin{array}{ccc}
1 & 0 & 0 \\
0 & \cos (i) & \sin (i) \\
0 & -\sin (i) & \cos (i)
\end{array}\right] \times\left[\begin{array}{ccc}
\cos \left(\Omega_{0}\right) & \sin \left(\Omega_{0}\right) & 0 \\
-\sin \left(\Omega_{0}\right) & \cos \left(\Omega_{0}\right) & 0 \\
0 & 0 & 1
\end{array}\right], \\
\mathbf{U}_{\mathbf{C}}(t)=\left[\begin{array}{ccc}
\cos \left(w_{e} \cdot t\right) & -\sin \left(w_{e} \cdot t\right) & 0 \\
\sin \left(w_{e} \cdot t\right) & \cos \left(w_{e} \cdot t\right) & 0 \\
0 & 0 & 1
\end{array}\right] .
\end{gathered}
$$




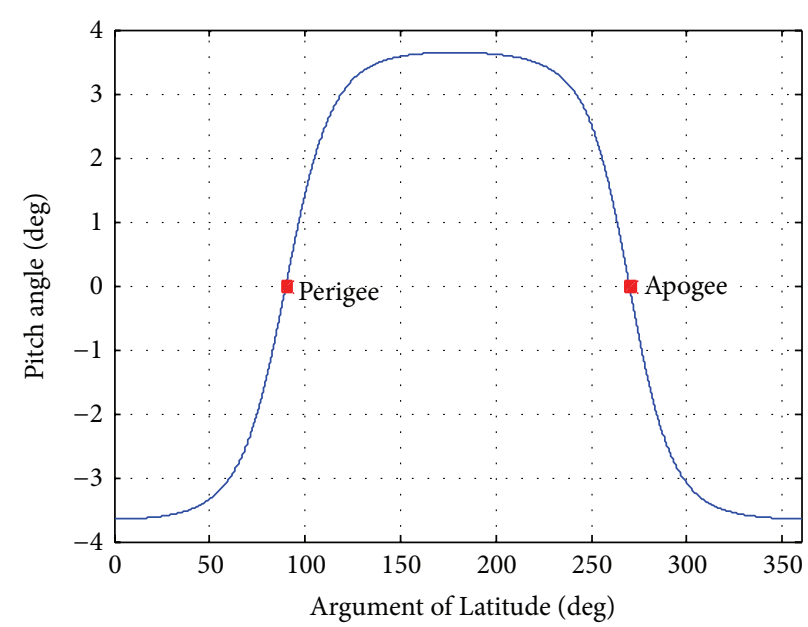

(a)

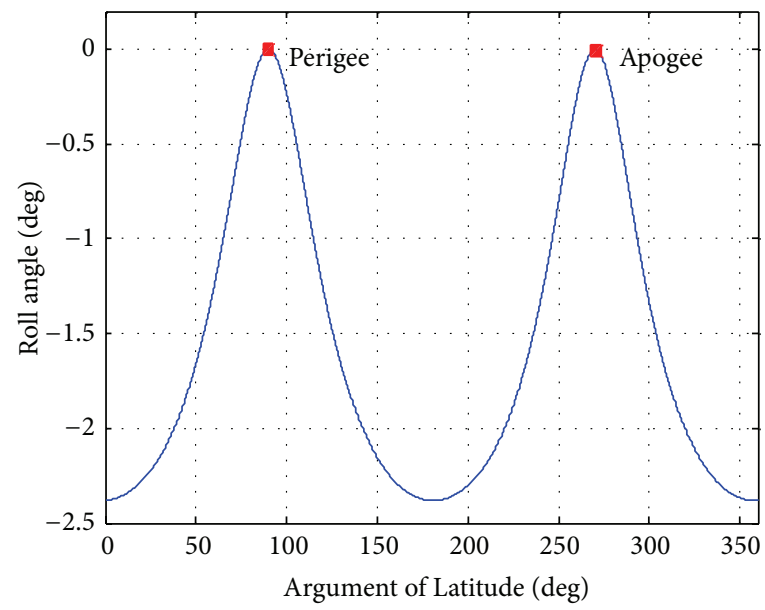

(b)

FIGURE 4: The pitch angles and the roll angles of the 2D method along the orbit.

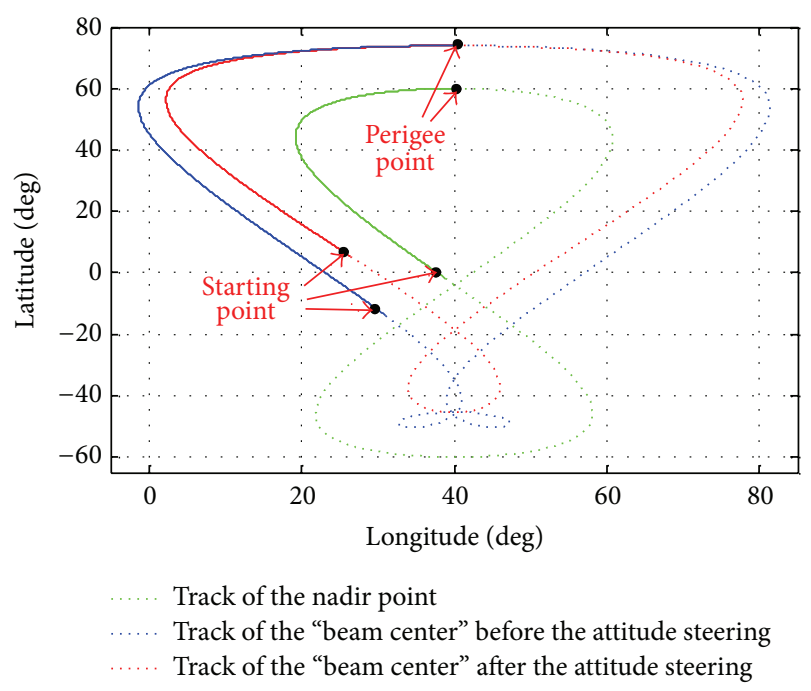

FIGURE 5: Comparisons of the track of the nadir point and two tracks of the BCP before and after the attitude steering.

Then, combining (4), the transformation matrixes between the track of the new BCP and the track of the original one in the SCS can be presented as

$$
\begin{aligned}
& {\left[\begin{array}{ccc}
1 & 0 & 0 \\
0 & \cos \left(\theta_{X}(t)\right) & -\sin \left(\theta_{X}(t)\right) \\
0 & \sin \left(\theta_{X}(t)\right) & \cos \left(\theta_{X}(t)\right)
\end{array}\right]} \\
& \quad \times\left[\begin{array}{ccc}
\cos \left(\theta_{Y}(t)\right) & 0 & \sin \left(\theta_{Y}(t)\right) \\
0 & 1 & 0 \\
-\sin \left(\theta_{Y}(t)\right) & 0 & \cos \left(\theta_{Y}(t)\right)
\end{array}\right] \\
& \times\left[\begin{array}{c}
R_{\mathrm{st}} \sin (\varphi) \cos (\theta) \\
-R_{\mathrm{st}} \sin (\varphi) \sin (\theta) \\
R_{\mathrm{st}} \cos (\varphi)
\end{array}\right]=\left[\begin{array}{l}
x_{0}(t) \\
y_{0}(t) \\
z_{0}(t)
\end{array}\right],
\end{aligned}
$$

where $\theta_{Y}(t)$ is the angle that the satellite rotates around the axis of $S y_{o}$ and $\theta_{X}(t)$ is the angle that the satellite rotates around the axis of $S x_{0}$. Based on the relationship between the attitude steering angles and the axis angles $\left(\theta_{X}(t)\right.$ and $\left.\theta_{Y}(t)\right)$ in (20), the pitch steering angle $\theta_{P}(t)$ and the roll steering angle $\theta_{R}(t)$ can be expressed as

$$
\begin{aligned}
\theta_{P}(t)=\arcsin & \left(\frac{x_{0}(t)}{R_{\mathrm{st}}(t)}\right)-\arcsin (\sin (\varphi) \cos (\theta(t)))-\psi(t), \\
\theta_{R}(t)= & -\arcsin \left(\frac{y_{0}(t)}{R_{\mathrm{st}}(t) \cdot \cos \left(\theta_{P}(t)\right)}\right) \\
& -\arcsin \left(\frac{\sin (\varphi) \sin (\theta(t))}{\sqrt{1-\sin ^{2}(\varphi) \cos ^{2}(\theta(t))}}\right) .
\end{aligned}
$$

\section{Simulation and Analysis}

For investigations of the numerically applied 2D steering method, the relevant orbit parameters of GEO SAR as listed in Table 1 are used.

Before the 2D attitude steering, the Doppler centroid $f_{\mathrm{dc}}$ varies along the orbit, as the blue line shown in Figure 3. The non-zero-Doppler centroid is caused by Earth's rotation and the elliptical orbit. The red line in Figure 3 shows that $f_{\mathrm{dc}}$ can be perfectly compensated to zero by using the approach presented in this paper.

In simulations, the GEO satellite starts at the ascending node, and the radar is left looking during the orbital period. Figure 4 depicts the two attitude angles of the proposed method which can minimize the Doppler centroid shifts. In Figure 4, the positive/negative values of the angle indicate the angles that the satellite rotates around its axis anticlockwise/ clockwise. It is apparent that both the attitude angles vary within a small range of degrees. The maximum pitch steering 


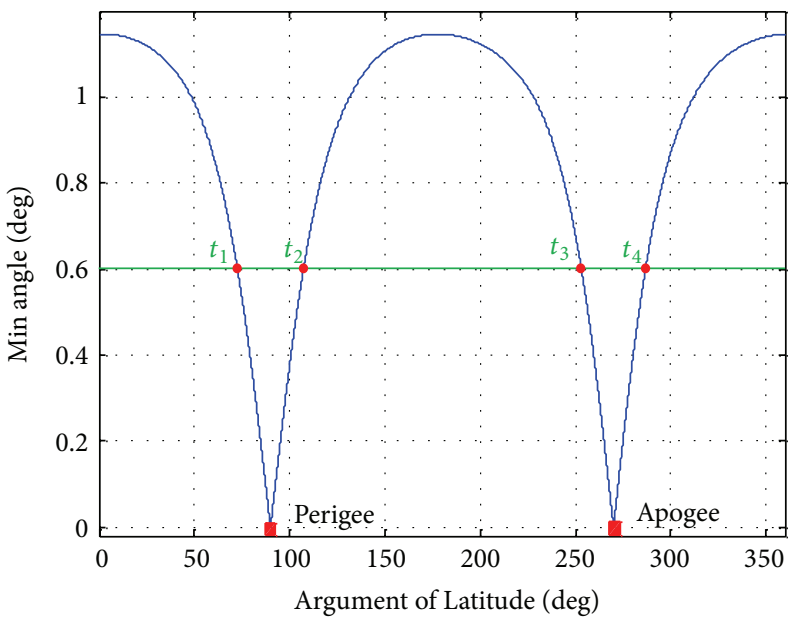

(a)

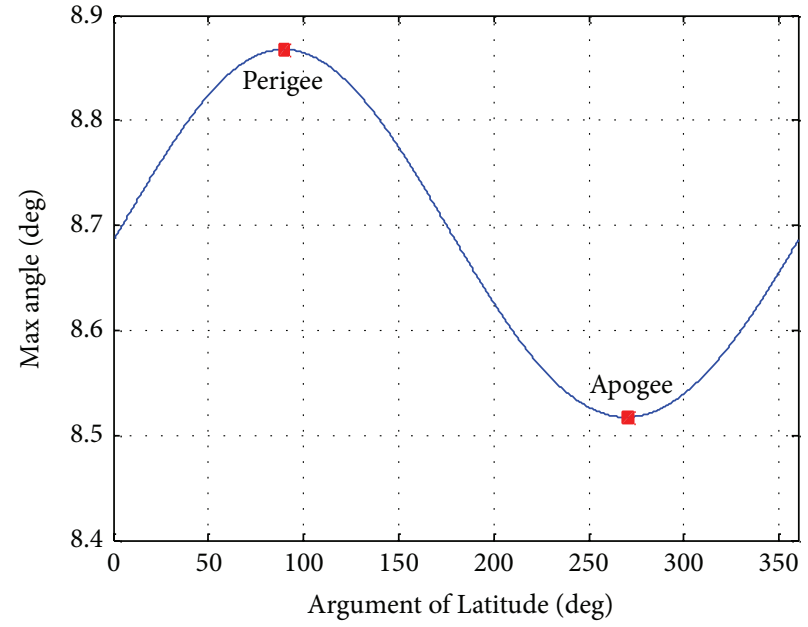

(b)

Figure 6: The effective look angles of the zero-Doppler centroid control along the orbit. (a) Minimum value of the look angle; (b) maximum value of the look angle.
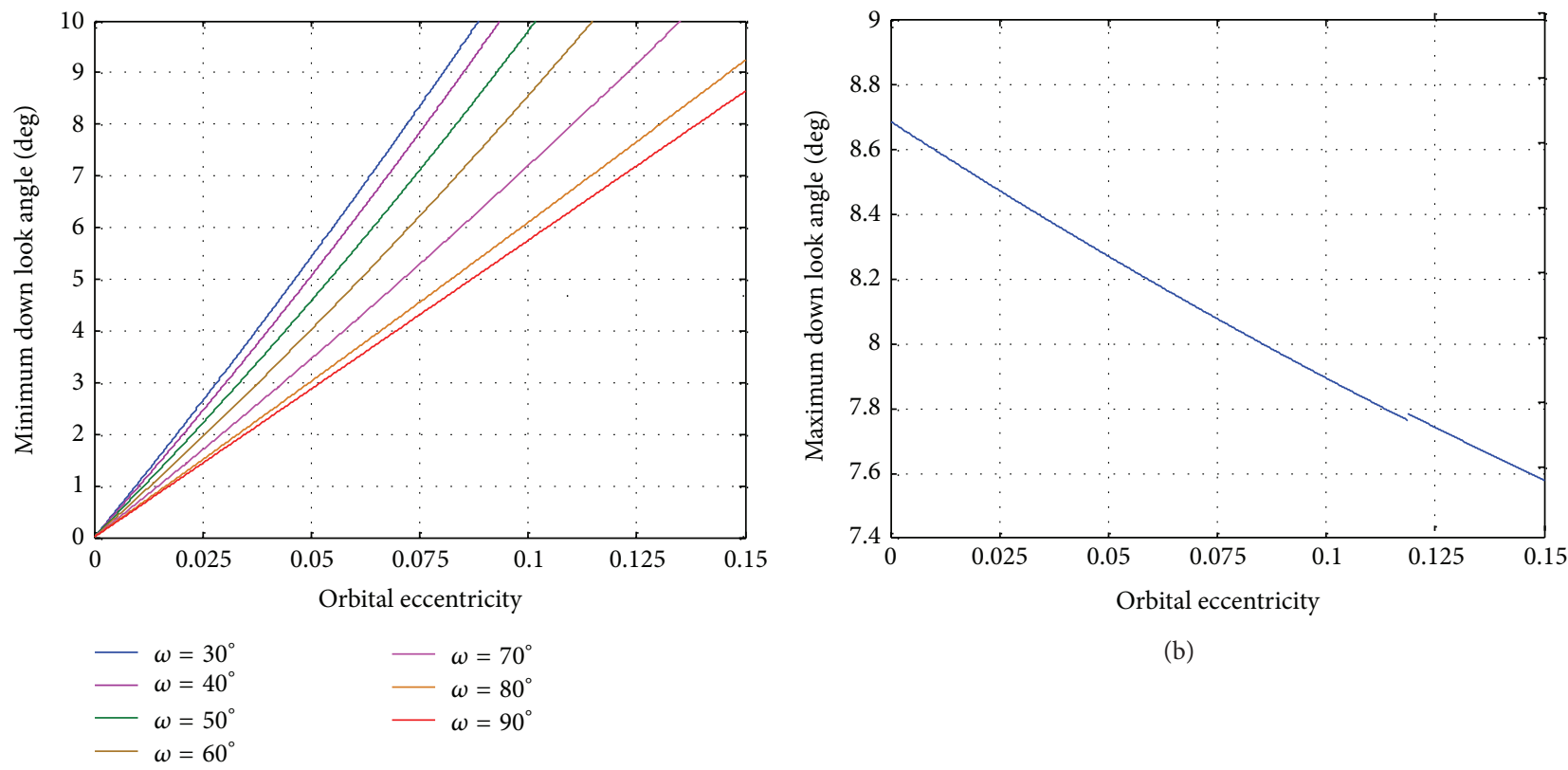

(b)

(a)

FIGURE 7: Relationship between the range of the look angle and the orbit parameters. (a) Minimum look angle variation with the eccentricity at different arguments of perigee; (b) Maximum look angle variation with the eccentricity.

angle is about $3.7^{\circ}$ and the maximum roll steering angle is nearly $2.4^{\circ}$. As can be seen, there is no demand on the attitude steering when the satellite platform lies in the perigee or apogee because the relative velocity of the satellite is perpendicular to the beam direction at that moment.

Figure 5 depicts the tracks of the BCP before and after the attitude steering and the track of the nadir point in the Earth's Latitude and Longitude Coordinate, represented by the red, blue, and green dotted lines. The red, blue, and green lines describe these three tracks (from the starting point to the perigee point) during the time that the satellite runs from the ascending node to the perigee.

As can be seen from Figure 5, there is a great difference between the red and blue lines at the starting point where the residual Doppler centroid is great. After that the red and blue lines come together at the perigee point, which means that there is no demand on the zero-Doppler centroid control when the satellite reaches its absolute perigee.

Figure 6 shows the effective range of the look angle $\varphi$ along the orbit. Specifically, the look angle should not be less 
TABLE 1: System parameters of GEO SAR.

\begin{tabular}{lc}
\hline Specification & Value \\
\hline Semimajor axis & $42220000 \mathrm{~m}$ \\
Orbit inclination & 60 degrees \\
Orbit eccentricity & 0.02 \\
Argument of perigee & 90 degrees \\
Right ascension of ascending node & 40 degrees \\
Look angle & 2.5 degrees \\
Beam width & 0.48 degrees \\
Signal wavelength & $0.25 \mathrm{~m}$ \\
Antenna diameter & $30 \mathrm{~m}$ \\
Pulse repetition frequency & $250 \mathrm{~Hz}$ \\
\hline
\end{tabular}

than the minimum angle (the blue line shown in Figure 6(a)) and not be greater than the maximum angle (shown in Figure 6(b)). If the look angle is $0.6^{\circ}$ after attitude steering, as the green line shown in Figure 6(a), the zero-Doppler centroid control is effective only during the time $\left[t_{1}, t_{2}\right]$ and $\left[t_{3}, t_{4}\right]$. Therefore, it is needed to take the effective range into account when the zero-Doppler centroid method is performed.

According to (17), the orbit parameters have a bearing on the range of look angle. Figure 7(a) illustrates the relationship between the minimum look angle and the orbital eccentricity at different arguments of perigee. There is an upward trend of the minimum look angle with the increase of eccentricity at all arguments of perigee. As the argument of perigee is $90^{\circ}$, the minimum value grows at the lowest speed, and it has the fastest growth rate when the argument of perigee is $60^{\circ}$. According to (16), the maximum value of the look angle only relates to $R_{t}$ and $R_{s}(t)$, and it decreases with increasing the orbital eccentricity (shown in Figure 7(b)). Generally, the minimum value is supposed to be less than the maximum one. Otherwise, the radar beam may radiate the space outside the Earth after the zero-Doppler centroid control, as in the case that the eccentricity is about 0.14 and $\omega_{0}=90^{\circ}$.

\section{Conclusion}

The contribution of this paper is that it introduces the effective range of the look angle, in which the zero-Doppler centroid control is feasible for GEO SAR system. Meanwhile, a new calculation approach is proposed for the attitude steering angles. The $2 \mathrm{D}$ method, combining the pitch steering with roll steering, provides a new implementation for the satellite attitude control. This approach is deduced from the aspect of the Earth observation rather than the satellite motion model. Therefore, it is suitable for general Earth orbit SAR system. Simulations validate the expressions derived and the proposed zero-Doppler centroid control method. Lastly, the relationship between the range of the look angle and the orbital parameters is illustrated, which would be helpful for the design of the satellite orbit.

\section{Conflict of Interests}

The authors declare that there is no conflict of interests regarding the publication of this paper.

\section{Acknowledgments}

This work was financially supported by the National Natural Science Foundation of China (61201308) and the sponsoring of the Aerospace Science and Technology Innovation Fund was acknowledged.

\section{References}

[1] X. Dong, Y. Gao, C. Hu, T. Zeng, and D. Chao, "Effects of Earth rotation on GEO SAR characteristics analysis," in Proceedings of the 6th International Conference on Radar (RADAR '11), vol. 1, pp. 34-37, October 2011.

[2] R. K. Raney, "Doppler properties of radars in circular orbit," International Journal of Remote Sensing, vol. 7, no. 9, pp. 1153$1162,1986$.

[3] E. Boerner, H. Fiedler, G. Krieger, and J. Mittermayer, "A new method for total zero doppler steering," in Proceedings of the IEEE International Geoscience and Remote Sensing Symposium (IGARSS '04), pp. 1526-1529, September 2004.

[4] H. Fiedler, E. Boerner, J. Mittermayer, and G. Krieger, “Total Zero Doppler steering: a new method for minimizing the Doppler centroid," IEEE Geoscience and Remote Sensing Letters, vol. 2, no. 2, pp. 141-145, 2005.

[5] Y. Ze, Z. Yinqing, C. Jie et al., "A new satellite attitude steering approach for zero Doppler centroid," in Proceedings of the IET International Radar Conference, pp. 1-4, April 2009.

[6] Y. J. Zhang, Y. S. Zhang, H. F. Huang, and Z. Dong, "Research on elliptic orbit total zero Doppler steering," in Proceedings of the 8th European Conference on Synthetic Aperture Radar (EUSAR '10), pp. 1-4, Aachen, Germany, 2010.

[7] X. Dong, Z. Ding, and Z. Zhao, "A method of zero Doppler centroid control," in Proceedings of the 8th European Conference on Synthetic Aperture Radar (EUSAR '10), pp. 1-4, VDE, 2010.

[8] T. Long, X. Dong, C. Hu, and T. Zeng, "A new method of zerodoppler centroid control in GEO SAR," IEEE Geoscience and Remote Sensing Letters, vol. 8, no. 3, pp. 512-516, 2011.

[9] L. J. Cantafio, Space-Based Radar Handbook, chapter 2, Artech House, 1989.

[10] T. Zeng, W. Yin, L. Zhao, and Z. Ding, "An improved velocity calculation model in GEO SAR system," in Proceedings of the IET International Radar Conference, pp. 1-5, 2013. 

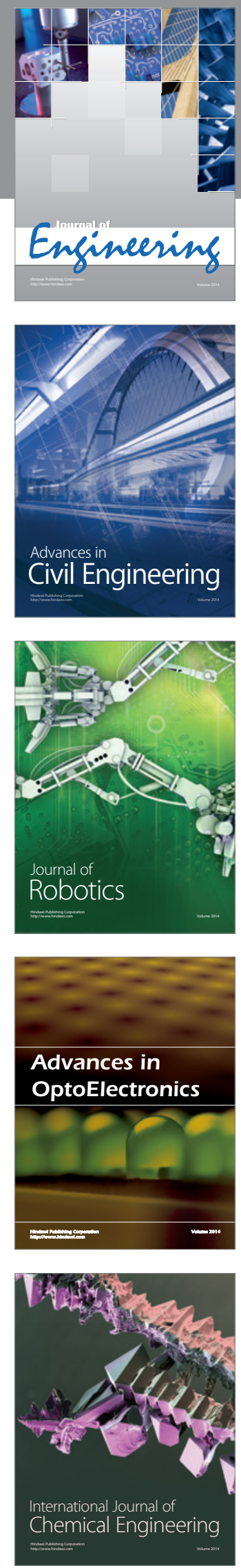

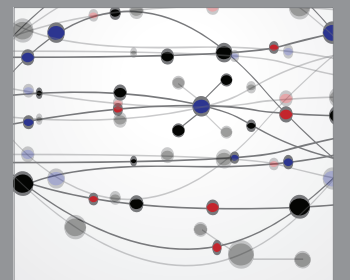

The Scientific World Journal
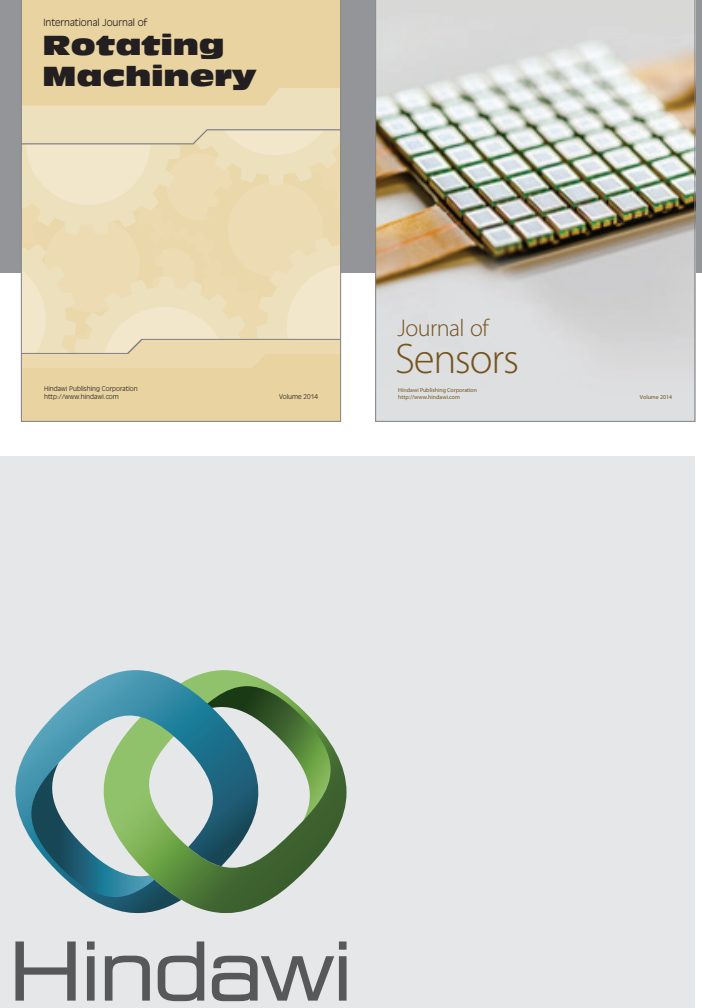

Submit your manuscripts at http://www.hindawi.com
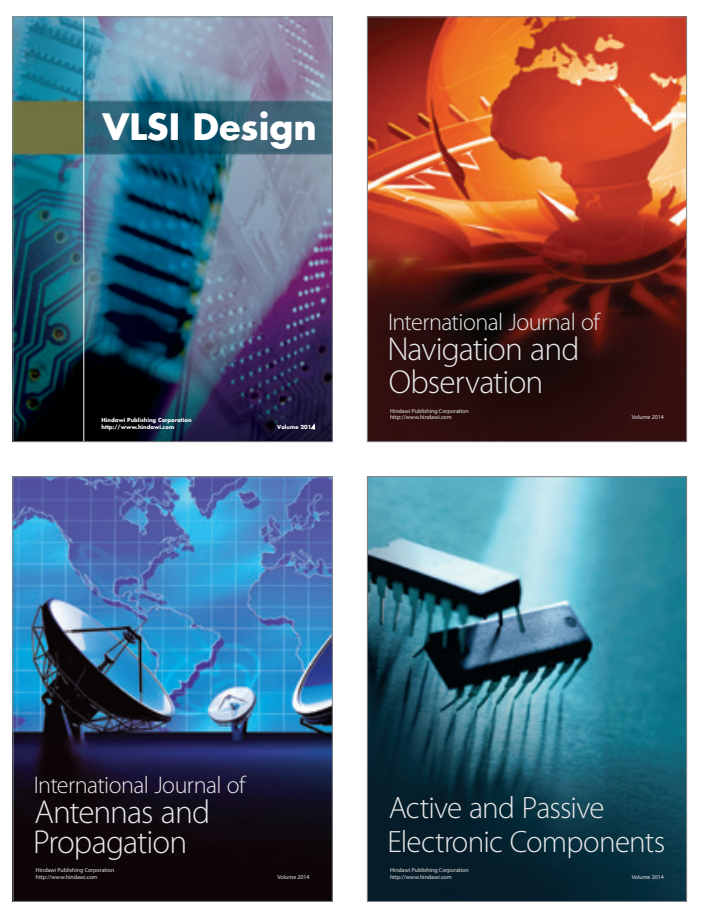
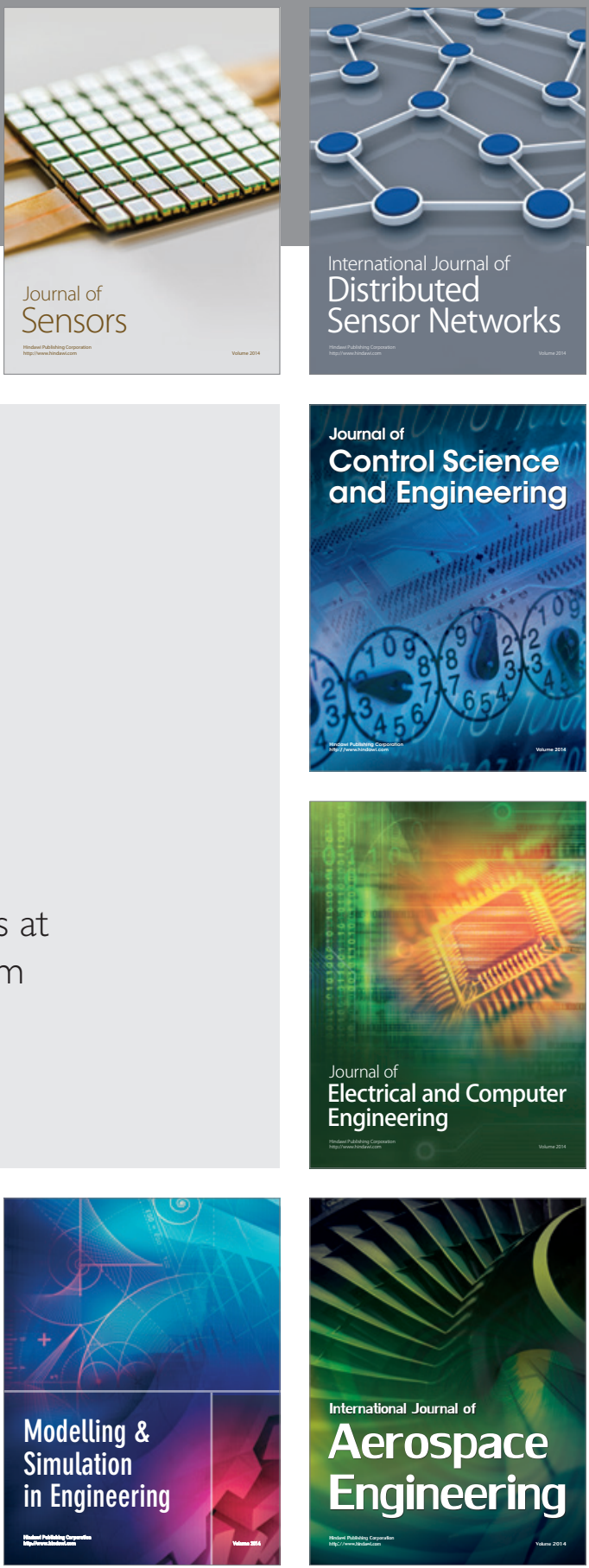

Journal of

Control Science

and Engineering
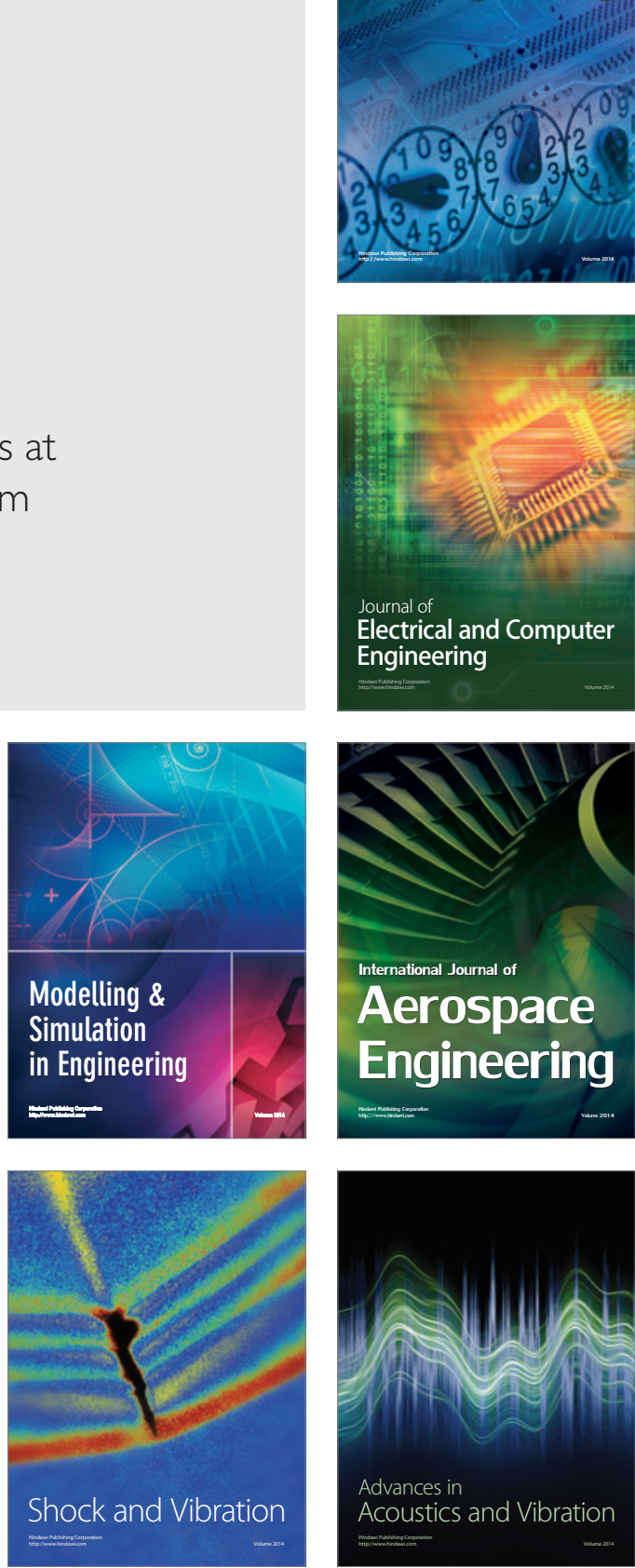